\title{
CARACTERIZAÇÃO DO ALGINATO PRODUZIDO POR Pseudomonas mendocina
}

\author{
RENATA LOPES DOS SANTOS* \\ RIVELI VIEIRA BIRGIDO** \\ ALFREDO TIBURCIO NUNES PIRES***

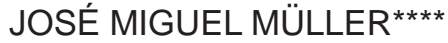

\begin{abstract}
O objetivo deste estudo foi avaliar a produção de alginato por Pseudomonas mendocina à partir dos substratos glicerol, sacarose e glicose. Nessa etapa do estudo comparou-se o rendimento de bioconversão e a produtividade do processo fermentativo, além das características físico-químicas dos materiais produzidos à partir dos substratos utilizados. Os melhores resultados em termos de rendimento e bioconversão foram observados quando o biopolímero foi produzido tendo sacarose como fonte de carbono. Observou-se que os biopolímeros produzidos apresentaram composição química similar e que as características reológicas do meio de cultura dependem da fonte de carbono utilizada.
\end{abstract}

PALAVRAS-CHAVE: ALGINATO BACTERIANO; BIOPOLÍMERO; Pseudomonas mendocina.

* Engenheira de Alimentos, Universidade Federal de Santa Catarina (UFSC), Florianópolis, SC (e-mail: renatals@gmail.com).

** Mestre em Engenharia de Alimentos, UFSC, Florianópolis, SC (e-mail: ribrigido@gmail.com).

*** Doutor em Química, Professor Adjunto, UFSC, Florianópolis, SC (e-mail: pires@qmc.ufsc.br).

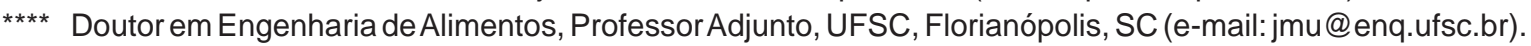




\section{INTRODUÇÃo}

O alginato, copolímero aniônico, é constituído por ácidos $\alpha$-L-gulurônicos e $\beta$-Dmanurônicos com ligações 1-4. O material varia extensamente em termos de sua proporção entre os resíduos manurônicos (M) e gulurônicos (G) (DRAGET, SKJÅK BRÆKK e SMIDSRØD, 1994). O alginato é extraído de algas marinhas da classe Phaeophyceae e sua estrutura sequencial e grau de polimerização dependem da espécie de alga e das condições do processo de extração.

$\mathrm{O}$ alginato tem sido amplamente utilizado em alimentos, cosméticos e medicamentos, encontrando aplicação nas indústrias têxtil e de papel. Também está sendo utilizado de forma inovadora nas áreas médica e farmacêutica (DRAGET e TAYLOR, 2009). Em função de suas características físicas e de sua capacidade de interagir com cátions pode ser empregado como espessante, estabilizante de emulsões e de espuma, agente de encapsulação, agente de gelificação, agente de formação de filmes e de fibras sintéticas, entre outras possibilidades.

Uma das propriedades apresentadas pelo alginato consiste na formação de geis na presença de cátions. Vários cátions são utilizados, sendo $\mathrm{Ca}^{2+} \mathrm{o}$ mais empregado. A estrutura formada, reportada como "caixa de ovos", resulta da interação dos cátions com os blocos gulurônicos. As propriedades finais dos geis formados dependem da composição e da sequência monomérica do alginato (DRAGET e TAYLOR, 2009).

O alginato apresenta boas características para formação de nanocápsulas (LERTSUTTHIWONG et al., 2008). Nanopartículas de alginato tem sido utilizadas na área farmacêutica e mostraram bom desempenho na liberação controlada de inibidores de proteases (ANGSHUMAN et al., 2010)e de insulina (SARMENTO et al., 2007).

$\mathrm{O}$ alginato pode ser sintetizado por Azotobacter vinelandii e Pseudomonas spp. O alginato microbiano difere do obtido de algas marinhas por apresentar grupos acetil ligados ao resíduo manurônico. Além do grau de acetilação, a composição monomérica e a massa molecular do biopolímero afetam fortemente as suas propriedades reológicas e físico-químicas. $\mathrm{O}$ alginato bacteriano pode ser produzido para fins específicos, com determinadas propriedades qualitativas, 0 que permite novas aplicações desse biopolímero (REHM, 2010).

O destaque para a produção de alginato por Pseudomonas mendocina deve-se ao fato da bactéria não apresentar patogenicidade para humanos, ao contrário do que ocorre com a Pseudomonas aeruginosa, e também por não precisar de controle tão rigoroso da tensão de oxigênio dissolvido durante o processo fermentativo, comparativamente ao Azotobacter vinelandii (SABRA, ZENG e DECKWER, 2001). A produção de biopolímeros por micro-organismos apresenta algumas vantagens, pois pode ser realizada sob condições controladas, usando-se substratos de qualidade constante que permitem a obtenção de materiais específicos com características uniformes.

A possibilidade de desenvolver processos ambientalmente amigáveis para produzir materiais biodegradáveis e, em alguns casos, materiais biocompatíveis torna-se cada vez mais atrativa para a indústria (REHM, 2010). Nesse contexto, a produção de alginato por micro-organismos à partir de substratos tradicionalmente utilizados em processos fermentativos ou de resíduos obtidos de processos industriais e agrícolas apresenta-se como alternativa a ser avaliada. Adicionalmente, sua produção por micro-organismos irá permitir a exploração controlada de suas fontes naturais (REMMINGHORST e REHM, 2006).

Considerando que a glicose tem sido a fonte de carbono utilizada na maioria dos estudos de produção de biopolímeros por micro-organismos, o objetivo deste trabalho foi avaliar adicionalmente outras fontes de carbono como o glicerol e a sacarose. Também foram avaliadas as características reológicas do meio de cultura obtido ao final do processo fermentativo à partir dos diferentes substratos. Os biopolímeros produzidos foram analisados por espectrometria de infravermelho. 


\section{MATERIAL E MÉTODOS}

\subsection{MICRO-ORGANISMO}

Utilizou-se o micro-organismo Pseudomonas mendocina M13 (CCT2245), mutante isolado pela técnica de resistência ao antibiótico carbenicilina (MÜLLER e MONTE ALEGRE, 2007).

\subsection{MEIO DE MANUTENÇÃO E PRODUÇÃO}

O meio empregado para manutenção do micro-organismo foi composto de (g.L-1): sacarose $(1,0), \mathrm{KH}_{2} \mathrm{PO}_{4}(0,3), \mathrm{Na}_{2} \mathrm{HPO}_{4}(0,5)$, peptona $(0,25)$ e $\mathrm{MgSO}_{4} \cdot 7 \mathrm{H}_{2} \mathrm{O}(0,25)$. O meio para produção do biopolímero, similar ao utilizado por Müller e Monte Alegre (2007), apresentava os seguintes constituintes (em g.L-1): glicose, sacarose ou glicerol (20, 40 ou 80), $\left(\mathrm{NH}_{4}\right)_{2} \mathrm{SO}_{4}(0,7)$, extrato de levedura $(0,1), \mathrm{MgSO}_{4} \cdot 7 \mathrm{H}_{2} \mathrm{O}(0,1), \mathrm{CaCl}_{2}(0,1) ; \mathrm{MnSO}_{4} \cdot 7 \mathrm{H}_{2} \mathrm{O}\left(0,86 \times 10^{-3}\right), \mathrm{ZnSO}_{4} \cdot 7 \mathrm{H}_{2} \mathrm{O}\left(0,2 \times 10^{-3}\right)$, $\mathrm{CoCl}_{2} \cdot 6 \mathrm{H}_{2} \mathrm{O}\left(0,28 \times 10^{-3}\right), \mathrm{FeSO}_{4} \cdot 7 \mathrm{H}_{2} \mathrm{O}\left(3,6 \times 10^{-3}\right) ; \mathrm{Na}_{2} \mathrm{HPO}_{4}(12,5) ; \mathrm{KH}_{2} \mathrm{PO}_{4}(3,0)$.

\subsection{CULTURAS EM FRASCOS AGITADOS}

Os meios de cultura, após inoculação, foram incubados em agitador rotatório a 240 rpm na temperatura de $30^{\circ} \mathrm{C}$ (MÜLLER e MONTE ALEGRE, 2007). Os ensaios foram realizados em triplicata.

\subsection{TÉCNICAS ANALÍTICAS}

Determinou-se a concentração de biomassa das amostras mediante leitura da densidade óptica (DO) a $600 \mathrm{~nm}$. Para a determinação da concentração de glicose utilizou-se kit colorimétrico marca "Gold Analisa". As enzimas glicose-oxidase e peroxidase atuam sobre a glicose, cuja concentração é proporcional ao composto de cor formado. Utilizou-se kit colorimétrico da mesma marca para determinar a concentração de glicerol, que sofre ação sequencial das enzimas glicerolquinase, G-3-P oxidase e peroxidase. A concentração de glicerol é proporcional ao composto de cor formado. Determinou-se a concentração de sacarose pelo método do ácido 3-5 dinitrossalicílico (DNS) (MILLER, 1959).

\subsection{BIOPOLÍMERO}

Efetuou-se a determinação da concentração do biopolímero pelo método gravimétrico, sendo o meio de cultura centrifugado a $18000 \mathrm{~g}$ por 20 minutos para separação das células. Ao sobrenadante foram adicionados três volumes de etanol para precipitar o biopolímero presente na amostra (SENGHA et al., 1989).

\subsection{CARACTERIZAÇAO REOLÓGICA E DE INFRAVERMELHO}

Realizou-se a caracterização reológica do meio de cultura com amostras do final do cultivo para os diferentes substratos utilizados em reômetro digital Brookfield. Os dados experimentais obtidos foram ajustados ao modelo de Ostwald-de-Waele $\left(\sigma=K Y^{n}\right)$, também conhecido como Lei da Potência, para determinação dos parâmetros reológicos $\mathrm{K}$ (índice de consistência) e $n$ (índice de comportamento) (STEFFE, 1996). As análises em infravermelho foram realizadas com os biopolímeros produzidos à partir dos substratos glicose, glicerol e sacarose em pastilhas de $\mathrm{KBr}$.

\subsection{CÁLCULO DA PRODUTIVIDADE E RENDIMENTO}

Para determinar a produtividade do biopolímero (Equação 1) considerou-se sua concentração ao final do cultivo: 


$$
P=\frac{m_{f}}{V \cdot t_{f}}
$$

Em que:

$P=$ produtividade em $\mathrm{g} \cdot \mathrm{L}^{-1} \cdot \mathrm{h}^{-1}, m_{f}=$ massa $(\mathrm{g})$ de alginato precipitado no instante final do cultivo, $V(\mathrm{~L})=$ volume da amostra e $t_{f}=$ tempo final (h).

O fator de conversão substrato em produto foi calculado para o final do cultivo utilizando-se a Equação 2:

Em que:

$$
Y_{p / s}=\frac{C_{f}-C_{i}}{S_{i}-S_{f}}
$$

$Y_{p / s}=$ fator de bioconversão de substrato em produto $\left(\mathrm{gP} \mathrm{gS}^{-1}\right), C_{f}=$ concentração de alginato precipitado no tempo final $\left(\mathrm{g} \cdot \mathrm{L}^{-1}\right), C_{i}=$ concentração de alginato no tempo inicial $\left(\mathrm{g} . \mathrm{L}^{-1}\right)$; $S_{i}=$ concentração de substrato no tempo inicial $\left(\mathrm{g} \cdot \mathrm{L}^{-1}\right)$, e $S_{f}=$ concentração de substrato no tempo final (g.L-1) (AQUARONE et al., 2001).

\section{RESULTADOS E DISCUSSÃO}

As melhores condições para produzir grandes quantidades de exo-polissacarídeos (EPS) por micro-organismos estão relacionadas a altos valores na relação de concentrações entre carbono e nitrogênio $[\mathrm{C} / \mathrm{N}]$. Ao esgotar a fonte de nitrogênio e tendo ainda disponível a fonte de carbono, o micro-organismo converte o excesso de carbono em biopolímero de reserva. A glicose, fonte de carbono usual em processos fermentativos, tem sido empregada na síntese de alginato por micro-organismos dos gêneros Pseudomonas e Azotobacter. Neste estudo foram testados também o glicerol e sacarose como fonte de carbono. $O$ interesse em avaliar esses materiais reside na possibilidade de utilizá-los para produzir biopolímeros com alto valor agregado. Testes prévios revelaram que o micro-organismo estudado mostrou-se hábil na metabolização do glicerol, indicando sua capacidade de utilizar a rota metabólica de neoglicogênese.

Meios de cultura contendo somente uma fonte de substrato foram utilizados com o objetivo de verificar o desempenho do micro-organismo em converter cada um deles em biopolímero. As condições experimentais foram estabelecidas tendo como referência ensaios prévios, cujos parâmetros indicaram bons rendimentos de bioconversão e produtividade. Para os experimentos, realizados em frascos agitados, foram utilizadas concentrações iniciais de 20,40 e 80 g.L-1 dos diferentes substratos avaliados.

$\mathrm{Na}$ Tabela 1 são apresentados os dados obtidos para os experimentos utilizando-se glicose como substrato, não sendo observada nas concentrações iniciais de 20 e 40 g.L. $\mathrm{L}^{-1}$ diferença significativa na produtividade do biopolímero. A maior produtividade alcançou $0,31 \mathrm{~g}^{\mathrm{L}} \mathrm{L}^{-1} \mathrm{~h}^{-1}$ para a concentração inicial de $20 \mathrm{~g} \cdot \mathrm{L}^{-1}$ de glicose e nessas condições obteve-se também o maior fator de bioconversão $(0,44$ gP.gS-1 $)$.

TABELA 1 - RESULTADOS REFERENTES A CULTURAS REALIZADAS COM GLICOSE COMO FONTE DE CARBONO PARA A PRODUÇÃO DE ALGINATO

\begin{tabular}{cccccc}
\hline$S_{o}$ & $S_{f}$ & $t$ & $C$ & $P$ & $Y_{p / s}$ \\
\hline 20 & $0,13 \pm 0,01$ & 28 & $8,80 \pm 1,05$ & $0,31 \pm 0,04^{\mathrm{a}}$ & $0,44 \pm 0,08$ \\
40 & $0,16 \pm 0,01$ & 38 & $11,35 \pm 0,41$ & $0,30 \pm 0,01^{\mathrm{a}}$ & $0,28 \pm 0,01$ \\
80 & $4,83 \pm 1,24$ & 84 & $13,28 \pm 1,00$ & $0,16 \pm 0,01^{\mathrm{b}}$ & $0,18 \pm 0,01$ \\
\hline
\end{tabular}

$S$ e $S_{f}=$ concentração inicial e final de glicose $\left(g . L^{-1}\right) ; t=$ tempo de cultivo $(h), C=$ concentração de alginato $\left(g \cdot L^{-1}\right), P=$ produtividade $\left(g \cdot \mathrm{L}^{-1} \mathrm{~h}^{-1}\right)$ e $\mathrm{Y}_{\mathrm{p} / \mathrm{s}}=$ fator de bioconversão $\left(\mathrm{gP} \cdot \mathrm{gS}^{-1}\right)$. Letras iguais na coluna indicam que não há diferença significativa entre as médias (Teste de Tukey, $p \leq 0,05$ ). 
$\mathrm{Na}$ Tabela 2 estão detalhados os resultados de culturas realizadas em diferentes concentrações de glicerol. As melhores condições de produção do biopolímero foram obtidas na concentração inicial de $20 \mathrm{~g} \cdot \mathrm{L}^{-1}$ de substrato. Nessas condições, o tempo de fermentação para

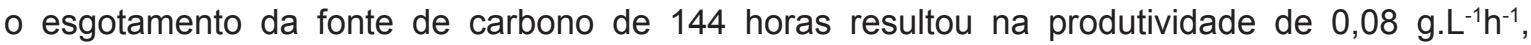
significativamente maior que a verificada nas concentrações de 40 e $80 \mathrm{~g} \cdot \mathrm{L}^{-1}$. Utilizando-se maiores concentrações de substrato (40 e 80 g.L-1) foram obtidos rendimentos menores.

\section{TABELA 2 - RESULTADOS REFERENTES A CULTURAS REALIZADAS COM GLICEROL COMO FONTE DE CARBONO PARA A PRODUÇÃO DE ALGINATO}

\begin{tabular}{cccccc}
\hline $\mathrm{S}_{0}$ & $\mathrm{~S}_{\mathrm{f}}$ & $\mathrm{t}$ & $\mathrm{C}$ & $\mathrm{P}$ & $\mathrm{Y}_{\mathrm{p} / \mathrm{s}}$ \\
\hline 20 & $0,11 \pm 0,01$ & 144 & $10,87 \pm 0,69$ & $0,08 \pm 0,01^{\mathrm{a}}$ & $0,55 \pm 0,03$ \\
40 & $0,08 \pm 0,04$ & 192 & $11,39 \pm 1,37$ & $0,06 \pm 0,01^{\mathrm{b}}$ & $0,29 \pm 0,03$ \\
80 & $12,94 \pm 0,69$ & 192 & $8,47 \pm 1,40$ & $0,04 \pm 0,01^{\mathrm{c}}$ & $0,13 \pm 0,02$ \\
\hline
\end{tabular}

$S_{\text {o }} S_{f}=$ concentração inicial e final de glicerol $\left(g \cdot L^{-1}\right) ; t=$ tempo de cultivo $(h), C=$ concentração de alginato $\left(g \cdot L^{-1}\right), P=$ produtividade $\left(g \cdot \mathrm{L}^{-1} \mathrm{~h}^{-1}\right)$ e $\mathrm{Y}_{\mathrm{p} / \mathrm{s}}=$ fator de bioconversão $\left(\mathrm{gP} \mathrm{gS} \mathrm{S}^{-1}\right)$. Letras iguais na coluna indicam que não há diferença significativa entre as médias (Teste de Tukey, $p \leq 0,05$ ).

Na Tabela 3 são apresentados os dados obtidos nos experimentos com sacarose como substrato. A maior quantidade de biopolímero produzida foi de $12,58 \mathrm{~g} \cdot \mathrm{L}^{-1}$ quando se utilizou a concentração inicial de $80 \mathrm{~g} \cdot \mathrm{L}^{-1}$ de sacarose. Pode-se observar também que o melhor rendimento de conversão de substrato em biopolímero, $0,41 \mathrm{gP}_{\mathrm{gS}}{ }^{-1}$, foi verificado na concentração de $20 \mathrm{~g} . \mathrm{L}^{-1}$. Considerando a produtividade, o melhor resultado foi de $0,17 \mathrm{~g} \cdot \mathrm{L}^{-1} \mathrm{~h}^{-1}$. Entretanto, observa-se que não há diferença significativa entre os ensaios realizados com as concentrações iniciais de substrato de 40 e 80 g.L - $^{-1}$.

\section{TABELA 3 - RESULTADOS REFERENTES A CULTURAS REALIZADAS COM SACAROSE COMO FONTE DE SUBSTRATO PARA A PRODUÇÃO DE ALGINATO}

\begin{tabular}{cccccc}
\hline$S_{o}$ & $S_{f}$ & $t$ & $C$ & $P$ & $Y_{p / s}$ \\
\hline 20 & $0,65 \pm 0,03$ & 72 & $7,89 \pm 0,61$ & $0,11 \pm 0,01^{\mathrm{a}}$ & $0,41 \pm 0,03$ \\
40 & $2,36 \pm 0,44$ & 72 & $10,99 \pm 1,23$ & $0,15 \pm 0,02^{\mathrm{b}}$ & $0,29 \pm 0,04$ \\
80 & $12,19 \pm 1,05$ & 72 & $12,58 \pm 1,81$ & $0,17 \pm 0,03^{\mathrm{b}}$ & $0,18 \pm 0,02$ \\
\hline
\end{tabular}

$\mathrm{S}_{\mathrm{o}}$ e $\mathrm{S}_{\mathrm{f}}=$ concentração inicial e final de glicerol $\left(\mathrm{g} \cdot \mathrm{L}^{-1}\right) ; \mathrm{t}=$ tempo de cultivo $(\mathrm{h}), \mathrm{C}=$ concentração de alginato $\left(\mathrm{g} \cdot \mathrm{L}^{-1}\right), \mathrm{P}=$ produtividade $\left(g \cdot \mathrm{L}^{-1} \mathrm{~h}^{-1}\right)$ e $\mathrm{Y}_{\mathrm{p} / \mathrm{s}}=$ fator de bioconversão $\left(\mathrm{gP} \mathrm{gS}^{-1}\right)$. Letras iguais na coluna indicam que não há diferença significativa entre as médias (Teste de Tukey, $p \leq 0,05$ ).

Avaliando-se o conjunto dos experimentos, as melhores condições de bioconversão foram obtidas na menor concentração (20 g.L-1 $)$ de todos os substratos utilizados.

Resultados reportados na literatura para a produção de alginato por Pseudomonas mendocina indicam rendimentos de $0,44 \mathrm{gP}_{\mathrm{gS}} \mathrm{g}^{-1}$, utilizando-se $20 \mathrm{~g} . \mathrm{L}^{-1}$ de glicose como substrato (MÜLLER e MONTE ALEGRE, 2007) e concentrações de $23,5 \mathrm{~g} \cdot \mathrm{L}^{-1}$ de alginato ao final do cultivo 
(SENGHA et al., 1989). Estudos de síntese de alginato por Azotobacter vinelandii tendo glicose como fonte de carbono resultaram na concentração do meio de $6,2 \mathrm{~g} \cdot \mathrm{L}^{-1}$, com rendimentos de bioconversão de $0,31 \mathrm{gP} \mathrm{gS}^{-1}$ (CHEN et al., 1985) e de 3,9 g.L-1 com rendimentos de bioconversão de 0,23 gP.gS $^{-1}$ (PARENTE et al., 1998).

$\mathrm{Na}$ produção de biopolímeros por micro-organismos observou-se mudança das características reológicas do meio de cultivo. Devido à liberação do biopolímero, durante o processo fermentativo, o meio de cultivo tornou-se mais espesso, apresentando características não newtonianas comuns a processos de produção de biopolímeros extracelulares.

Para o conjunto de experimentos realizados, amostras coletadas ao final do cultivo tiveram as suas características reológicas avaliadas. Os dados reológicos obtidos foram ajustados ao modelo de Ostwald-de-Waele. Os parâmetros $\mathrm{K}$ = índice de consistência e $n$ - índice de comportamento são reportados na Tabela 4.

\section{TABELA 4 - PARÂMETROS DE AJUSTE DO MODELO DE OSTWALD-DE-WAELE (LEI DA POTÊNCIA) PARA OS MEIOS DE CULTURA AO FINAL DO PROCESSO FERMENTATIVO}

\begin{tabular}{cccc|ccc|cccc}
\hline \multicolumn{4}{c|}{} & \multicolumn{3}{c|}{ Glicerol } & \multicolumn{3}{c}{ Sacarose } \\
\hline $\mathrm{S}$ 。 & $\mathrm{C}\left(\mathrm{g} \cdot \mathrm{L}^{-1}\right)$ & $n$ & $\mathrm{~K}\left(\mathrm{mPa}^{\mathrm{n}}\right)$ & $\mathrm{C}\left(\mathrm{g} \cdot \mathrm{L}^{-1}\right)$ & $n$ & $\mathrm{~K}\left(\mathrm{mPa}^{\mathrm{n}}\right)$ & $\mathrm{C}\left(\mathrm{g} \cdot \mathrm{L}^{-1}\right)$ & $n$ & $\mathrm{~K}\left(\mathrm{mPa}^{\mathrm{n}}\right)$ \\
20 & 9,50 & 0,57 & 202,8 & 11,74 & 0,65 & 106,90 & 7,50 & 0,41 & 1116,8 \\
40 & 11,26 & 0,66 & 59,02 & 10,32 & 0,70 & 45,50 & 12,91 & 0,39 & 1659,6 \\
80 & 14,56 & 0,61 & 90,99 & 8,19 & 0,58 & 20,99 & 19,36 & 0,42 & 1472,3 \\
\hline
\end{tabular}

$\mathrm{S}_{\mathrm{o}}=$ concentração inicial $\left(\mathrm{g} \cdot \mathrm{L}^{-1}\right) ; \mathrm{C}=$ concentração de alginato $\left(\mathrm{g} \cdot \mathrm{L}^{-1}\right) ; n=$ índice de comportamento; $\mathrm{K}=$ índice de consistência $\left(\mathrm{mPa}^{\mathrm{n}}\right)$.

Todos os meios de cultura ao final do processo fermentativo apresentaram comportamento pseudoplástico, ou seja, as viscosidades aparentes em todas as concentrações analisadas decresceram com o aumento da taxa de deformação. A comparação entre o índice de comportamento (n) das amostras indicou que o meio de cultura obtido com sacarose como substrato foi o que mais se afastou do comportamento newtoniano, pois apresentou os menores valores de $n$. Os valores mais altos para o índice de consistência $(K)$ foram também apresentados pelo meio de cultura tendo sacarose como substrato. Os meios de cultura obtidos à partir da glicose e do glicerol mostraram valores maiores de $n$ e menores de K.

Os biopolímeros sintetizados foram também avaliados por espectroscopia em infravermelho para verificar o efeito dos substratos utilizados sobre sua composição química. Como pode ser observado nos espectrogramas em infravermelho das amostras obtidas ao final do processo fermentativo (Figura 1) não há diferença entre os materiais sintetizados à partir dos diferentes substratos. As bandas de absorção nos comprimentos de onda de $1720 \mathrm{~cm}^{-1}$ e $1257 \mathrm{~cm}^{-1}$ indicam que o biopolímero é acetilado. Em alginatos produzidos por micro-organismos, o grupo acetil está associado exclusivamente aos resíduos manurônicos e sendo necessário pode ser facilmente removido. O espectro obtido mostrou-se similar aos apresentados por Hacking et al. (1983) e Müller e Monte Alegre (2007). 


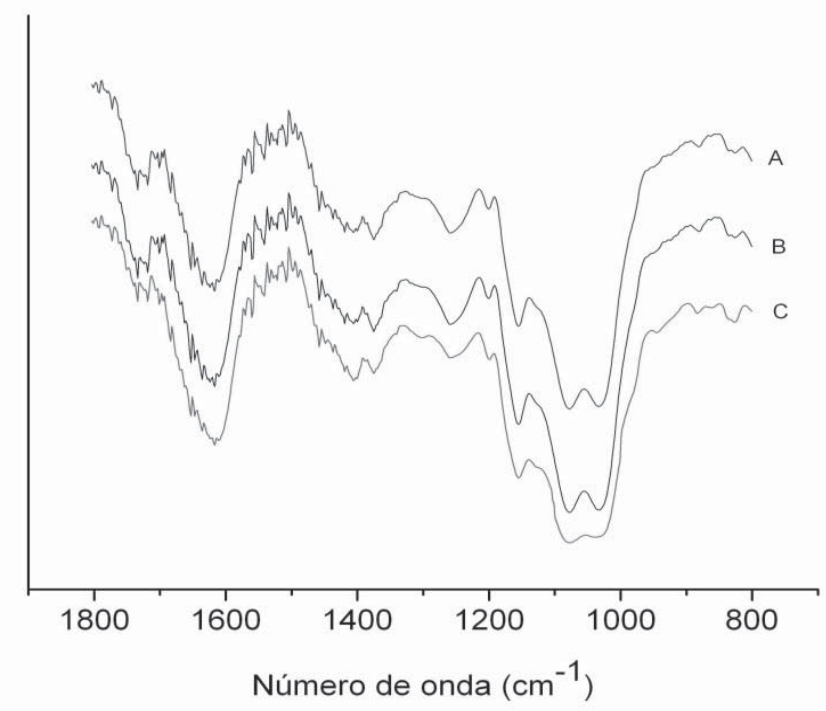

\section{FIGURA 1 - ESPECTRO INFRAVERMELHO DOS MATERIAIS BIOSSINTETIZADOS POR Pseudomonas mendocina UTILIZANDO COMO SUBSTRATO:} (A) GLICOSE; (B) SACAROSE E (C) GLICEROL

\section{CONCLUSÃO}

Os resultados obtidos nos experimentos mostram que maiores concentrações de substrato não resultaram em maiores concentrações de biopolímero ao final do processo fermentativo, condição que pode estar associada à limitação de nutrientes durante o processo fermentativo. Dentre os biopolímeros produzidos com os diferentes substratos, o material obtido a partir da sacarose apresentou o maior índice de consistência e as características pseudoplásticas mais pronunciadas (menor valor de $n$ ).

A composição do biopolímero não é afetada pelo uso de diferentes substratos, entretanto outras características como grau de acetilação e massa molecular, que podem estar relacionadas aos diferentes comportamentos reológicos observados nos meios de cultura obtidos, precisam ser avaliadas em novos experimentos.

A produção de alginato por micro-organismos irá permitir a obtenção de materiais com alto valor agregado a partir de matérias-primas já empregadas em processos fermentativos tradicionais como a sacarose ou na utilização de resíduos agroindustriais que é o caso do glicerol obtido na produção de biodiesel.

\section{ABSTRACT}

\section{CHARACTERIZATION OF ALGINATE PRODUCED BY Pseudomonas mendocina}

The purpose of this study was to evaluate the production of alginate by Pseudomonas mendocina from substrates: glycerol, sucrose and glucose. At this stage of the study were compared the yield of bioconversion and the productivity of fermentation process, as well as physicochemical characteristics the materials produced from the different used substrates. The best results in terms of yield and bioconversion were observed when the polymer was produced with sucrose as carbon source. It was observed that the polymers produced had similar chemical compositions and the rheological characteristic of the culture medium depended on the carbon source used.

KEY-WORDS: BACTERIAL ALGINATE; BIOPOLYMER; Pseudomonas mendocina. 


\section{REFERÊNCIAS}

1 ANGSHUMAN, B.; BHADTTACHARJEE, S. K.; MAHANTA, R.; BISWANATH, M.; BANDYOPADHAYAY, S. K. Alginate based nanoparticulate drug delivery for anti HIV drug Lopinavir. Journal of Global Pharma Technology, v.2, p. 126-132, 2010.

2 AQUARONE, E.; BORZANI, W.; SCHMIDELL, W.; LIMA, U. A. Biotecnologia industrial: biotecnologia da produção de alimentos. São Paulo: Edgard Blucher, 2001. 544 p.

3 CHEN, W. P.; CHEN, J. Y.; CHAN, S. C.; SU, C. L. Bacterial alginate produced by a mutant of Azotobacter vinelandii. Applied and Environmental Microbiology, v.49, n.3, p.543-546, 1985.

4 DRAGET, K. I.; SKJÅK BRÆKK, G.; SMIDSRØD, O. Alginic acid gels: the effect of alginate chemical composition and molecular weight. Carbohydrate Polymers, v. 25, p. 31-38, 1994.

5 DRAGET, K.; TAYLOR, C. Chemical, physical and biological properties of alginates and their biomedical implications. Food Hydrocolloids, v. 23, p.251-256, 2009.

6 HACKING, A. J.; TAYLOR, W. F.; JARMAN, T. R.; GOVAN, J. R. W. Alternative pathways for the biosynthesis of alginate from fructose and glucose in P.mendocina and A. vinelandii. Journal of General Microbiology, v.129, p. 1045-1052, 1983.

7 LERTSUTTHIWONG, P.; NOOMUN, K.; JONGAROONNGAMSANG, N.; ROJSITTHISAK, P.; NIMMANNIT, U. Preparation of alginate nanocapsules containing turmeric oil. Carbohydrate Polymers, v.74, p.209-214, 2008.

8 MILLER, G. L. Use of dinitrosalicylic acid reagent for determination of reducing sugar. Analytical Chemistry, v. 31, p. 426-428, 1959.

9 MÜLLER, J. M.; MONTE ALEGRE, R. Alginate production by P. mendocina in a stirred draft fermenter. World Journal of Microbiology and Biotechnology, v.23, p. 691-695, 2007.

10 PARENTE, E.; CRUDELE, M.A.; AQUINO, M.; CLEMENTI, F. Alginate production by Azotobacter vinelandii DSM576 in batch fermentation. Journal of Industrial Microbiology and Biotechnology, v.20, p.171-176, 1998.

11 REHM, B. H. A. Bacterial polymers: biosynthesis, modifications and applications. Nature - Applied Microbiology and Biotechnology, v. 8, p. 578-592, 2010.

12 REMMINGHORST, U.; REHM, B. H. A. Bacterial alginates: from biosynthesis to applications. Biotechnology Letters, v.28, p.1701-1712, 2006.

13 SABRA, W.; ZENG, A.P.; DECKWER, W.D. Bacterial alginate: physiology, product quality and process aspects. Applied Microbiology and Biotechnology, v. 56, p. 315-325, 2001.

14 SARMENTO, B.; RIBEIRO, A. J.; VEIGA, F.; FERREIRA, D.C.; NEUFELD, R. J. Insulin-loaded nanoparticles are prepared by alginate ionotropic pre-gelation followed by chitosan polyelectrolyte complexation. Journal of Nanoscience and Nanotechnology, v.7, p. 2833-2841, 2007.

15 SENGHA, S. S.; ANDERSON, A. J.; HACKING, A. J.; DAWES, E. A. The production of alginate by Pseudomonas mendocina in batch and continuous culture. Journal of General Microbiology, v.1135, p.795-804, 1989.

16 STEFFE, J. F. Rheological methods in food process engineering. Miami: Freeman Press, 1996. 418 p. 